Check for updates

Cite this: RSC Adv., 2020, 10, 13517

Received 15th January 2020

Accepted 6th March 2020

DOI: 10.1039/d0ra00449a

rsc.li/rsc-advances

\section{In situ synthesis of MWCNT-graft-polyimides: thermal stability, mechanical property and thermal conductivity}

\author{
Chunbo Wang, Bing Cong, Junyu Zhao, Xiaogang Zhao, DD Daming Wang, \\ Hongwei Zhou and Chunhai Chen (iD)*
}

\begin{abstract}
Herein, MWCNT-graft-polyimides (MWCNT-g-PIs) were prepared by the in situ grafting method. Strengthening the interfacial interaction between MWCNTs and polyimide chains decreased their interfacial thermal resistance $\left(R_{\mathrm{C}}\right)$. In contrast to the $R_{\mathrm{C}}$ of $10 \% \mathrm{MWCNT} / \mathrm{PIs}$, the $R_{\mathrm{C}}$ of $10 \% \mathrm{MWCNT}-\mathrm{g}$-PI decreased by $16.7 \%$. Hence, MWCNT-g-Pls possessed higher thermal conductivity than MWCNT/ polyimides (MWCNT/PIs). Meanwhile, the $T_{\mathrm{g}}$ values of all the samples (MWCNT/PIs and MWCNT-g-PIs) were greater than $399^{\circ} \mathrm{C}$ (by DMA). Compared with MWCNT/PIs, 5\% and 10\% MWCNT-g-PIs showed enhancement in thermal stability in air. The storage modulus retentions were greater than $63 \%$ at $200{ }^{\circ} \mathrm{C}$ and $45 \%$ at $300{ }^{\circ} \mathrm{C}$. Also, $5 \%$ and $10 \%$ MWCNT-g-PIs maintained the high tensile strength of pure PI, and the tensile modulus increased up to $2.59 \mathrm{GPa}$ on increasing the loading amount of MWCNTs. This study sheds light on improving the thermal conductivity of polyimides effectively at relatively low loadings.
\end{abstract}

\section{Introduction}

In recent years, with the rapid development of highperformance microelectronic equipment and energy harvesting devices, the demand for heat sinks in industrial and electronic fields has dramatically increased. ${ }^{\mathbf{1 , 2}}$ However, the thermal conductivity of common polymers is quite low and ranges from $0.1 \mathrm{~W} \mathrm{~m}^{-1} \mathrm{~K}^{-1}$ to $0.3 \mathrm{~W} \mathrm{~m}^{-1} \mathrm{~K}^{-1}$. Hence, their applications are severely limited in industrial and electronic fields due to heat accumulation. $^{3-5}$

It is important to increase the thermal conductivity of polymers to enhance the thermal diffusion and then reduce the heat accumulation. A simple and feasible method for enhancing the thermal conductivity of polymers involves introducing highly thermally conductive fillers (carbon nanotubes, ${ }^{\mathbf{4}, \mathbf{6}, 7}$ graphites, ${ }^{8-10}$ boron nitrides, ${ }^{11,12}$ aluminum nitrides, ${ }^{13,14}$ and aluminum oxides $^{\mathbf{1 5}}$ ) into polymers.

Among all kinds of highly thermally conductive fillers, carbon nanotubes (MWCNTs or SWCNTs) have been expected to be capable of improving the thermal conductivity of polymers effectively at relatively low loadings. ${ }^{\mathbf{1 6 - 1 9}}$ However, the poor thermal conductive performances of carbon nanotube composites are due to the high interfacial thermal resistance between carbon nanotubes and polymers.

Key Laboratory of High Performance Plastics (Jilin University), Ministry of Education, National \& Local Joint Engineering Laboratory for Synthesis Technology of High Performance Polymer, College of Chemistry, Jilin University, Changchun, 130012, P. R. China.E-mail: cch@jlu.edu.cn
Improving the filler/polymer interfaces can reduce "thermal resistance", and some methods have also been considered, such as non-covalent functionalization ${ }^{7}$ and covalent functionalization. ${ }^{6}$ Covalent functionalization involves grafting some chemical functional groups (amines, silanes, polymers, etc.) onto carbon nanotubes.

In this paper, polyimide was selected as a polymer matrix owing to its outstanding thermal and mechanical properties and MWCNTs acted as thermally conductive fillers. MWCNTgraft-polyimides (MWCNT-g-PIs) were obtained by the in situ grafting method for reducing the interfacial thermal resistance between nanotubes and polyimide to enhance the thermal conductivity. The thermal stability, mechanical properties and thermal conductivity of MWCNT-g-PIs were studied. For comparison, MWCNT/polyimides (MWCNT/PIs) were prepared by a simple blending method.

\section{Experimental}

\subsection{Materials}

MWCNTs (OD: 8-15 nm, length: $0.5-2 \mu \mathrm{m}$, purity $>98 \%$ ) and MWCNT-OHs (OD: 8-15 nm, length: $0.5-2 \mu \mathrm{m}$, purity $>98 \%$, -OH content: $3.06 \mathrm{wt} \%$ ) were bought from Chengdu Organic Chemicals Co., Ltd. Chinese Academy of Sciences. Pyromellitic dianhydride (PMDA) supplied by Sinopharm Chemical Reagent Beijing Co. Ltd was dried in vacuum at $200{ }^{\circ} \mathrm{C}$ for $10 \mathrm{~h}$ prior to use. 4,4'-Diaminodiphenyl ether $\left(4,4^{\prime}\right.$-ODA $)$ also supplied by Sinopharm Chemical Reagent Beijing Co., Ltd was dried in vacuum at $80{ }^{\circ} \mathrm{C}$ for $10 \mathrm{~h}$ prior to use. (3-Aminopropyl)triethoxysilane (APTES) supplied by Aladdin Reagent Co., Ltd was 
used without further purification. N,N-Dimethylacetamide (DMAc) was purified by vacuum distillation and stored in a bottle in the presence of $4 \AA$ molecular sieves prior to use.

\subsection{Measurements}

FTIR spectra were recorded on a Nicolet iS10 spectrometer at a resolution of $2 \mathrm{~cm}^{-1}$ in the range of $400-4000 \mathrm{~cm}^{-1}$ with reflection mode. Dynamic Mechanical Analysis (DMA) was performed with a TA instrument (DMA Q800) at the heating rate of $5{ }^{\circ} \mathrm{C} \min ^{-1}$ and a load frequency of $1 \mathrm{~Hz}$ in the film tension geometry and $T_{\mathrm{g}}$ was regarded as the peak temperature of $\tan \delta$ curves. Thermogravimetric analysis (TGA) was performed with the TA instrument 2050, with a thermal heating rate of $10{ }^{\circ} \mathrm{C} \mathrm{min}^{-1}$ in nitrogen or air atmosphere. The mechanical properties of the samples were studied at room temperature by a Shimadzu AG-I universal testing apparatus with a crosshead speed of $2 \mathrm{~mm} \mathrm{~min}^{-1}$. Measurements were obtained at $25{ }^{\circ} \mathrm{C}$ with film specimens (about $50 \mu \mathrm{m}$ thick, $6 \mathrm{~mm}$ wide and $40 \mathrm{~mm}$ long). The cross-section morphology of films was observed by Scanning Electron Microscopy (SEM, NOVA NANOSEM 450, England). The films were fractured in liquid nitrogen and coated with gold prior to test. Thermal conductivity measurements were performed at $25{ }^{\circ} \mathrm{C}$ by thermal conductivity instrument of TC 3000 series based on ASTM D5930 Standard Test Method for Thermal Conductivity of Plastics by means of a Transient Line Source Technique. Thermal conductivity $K(\mathrm{~W}$ $\mathrm{m}^{-1} \mathrm{~K}^{-1}$ ) was calculated by the following equation:

$$
K=\frac{q}{4 \pi(d \Delta T / d \ln t)}
$$

Here, $q$ represents the heat conducted per unit length of the wire, $\Delta T$ represents the temperature changes in the wire and $t$ represents the measuring time.

\subsection{Material preparations}

2.3.1 Synthesizing MWCNT/PIs via blending method. Samples with different MWCNT contents (0\%, 5\%, and 10\%) in polyimide were synthesized via the blending method and designated as PI, 5\% MWCNT/PI, and 10\% MWCNT/PI, respectively. The preparation of $5 \%$ MWCNT/PI was used as a representative to illustrate the detailed synthetic procedure. First, $0.2202 \mathrm{~g}$ MWCNTs and $25 \mathrm{~g}$ DMAc were added into a three-neck flask and then, the mixture was subjected to ultrasonic dispersion at room temperature for $3 \mathrm{~h}$. Subsequently, ODA (10 mmol, $2.002 \mathrm{~g}$ ), PMDA (10 mmol, $2.181 \mathrm{~g}$ ), and $14.6 \mathrm{~g}$ DMAc were added into the three-neck flask. The reaction mixture was slowly stirred for $24 \mathrm{~h}$. Next, the mixture was casted on a glass plate, followed by a preheating program $\left(60^{\circ} \mathrm{C} / 10 \mathrm{~h}\right.$, $80{ }^{\circ} \mathrm{C} / 2 \mathrm{~h}, 100{ }^{\circ} \mathrm{C} / 2 \mathrm{~h}, 120{ }^{\circ} \mathrm{C} / 2 \mathrm{~h}$ ) and an imidization procedure under vacuum $\left(200{ }^{\circ} \mathrm{C} / 1 \mathrm{~h}, 250{ }^{\circ} \mathrm{C} / 1 \mathrm{~h}\right.$, and $\left.300{ }^{\circ} \mathrm{C} / 1 \mathrm{~h}\right)$ to produce the $5 \% \mathrm{MWCNT} / \mathrm{PI}$ film.

2.3.2 Preparing MWCNT-g-PIs by in situ grafting method (Scheme 1). The different MWCNT contents (0\%, 5\%, and 10\%) were grafted on polyimide via the in situ synthesis method and the corresponding samples were named $g$-PI, 5\% MWCNT- $g$-PI, and $10 \%$ MWCNT- $g$-PI. The preparation of 5\% MWCNT- $g$-PI was used as a representative to illustrate the detailed synthetic procedure. First, $0.2204 \mathrm{~g}$ MWCNT-OH and $25 \mathrm{~g}$ DMAc were added into a three-neck flask and then, the mixture was subjected to ultrasonic dispersion at room temperature for $3 \mathrm{~h}$. Subsequently, ODA (9.8 mmol, 1.962 g), PMDA (10 mmol, 2.181 $\mathrm{g})$, and $14.7 \mathrm{~g}$ DMAc were added into the three-neck flask. The reaction mixture was slowly stirred for $2 \mathrm{~h}$. At last, APTES ( $0.2 \mathrm{mmol}, 0.0443 \mathrm{~g}$ ) was introduced into the system, and the system underwent polymerization for $24 \mathrm{~h}$. Then, the mixture was casted on a glass plate, followed by a preheating program $\left(60{ }^{\circ} \mathrm{C} / 10 \mathrm{~h}, 80{ }^{\circ} \mathrm{C} / 2 \mathrm{~h}, 100{ }^{\circ} \mathrm{C} / 2 \mathrm{~h}, 120{ }^{\circ} \mathrm{C} / 2 \mathrm{~h}\right)$ and an imidization procedure under vacuum $\left(200{ }^{\circ} \mathrm{C} / 1 \mathrm{~h}, 250{ }^{\circ} \mathrm{C} / 1 \mathrm{~h}\right.$, and 300 ${ }^{\circ} \mathrm{C} / 1 \mathrm{~h}$ ) to produce the $5 \%$ MWCNT- $g$-PI film.

\section{Results and discussion}

\subsection{Characterization of MWCNT/PIs and MWCNT- $g$-PIs}

Fig. 1 demonstrates the dispersed state of carbon nanotubes in DMAc. Fig. 1(B) shows the dispersed state after sonicating for $3 \mathrm{~h}$; this indicated that MWCNTs and MWCNT-OH were equally dispersed in DMAc and stood for $12 \mathrm{~h}$ and $24 \mathrm{~h}$, respectively, after sonication without sedimenting towards the bottom of the bottle evidently (Fig. 1(C) and (D)). Hence, DMAc was selected as the solvent to disperse carbon nanotubes and synthesize MWCNT/PIs and MWCNT- $g$-PIs.

The chemical structures of MWCNT- $g$-PIs were characterized by FT-IR spectroscopy. Fig. 2 demonstrates the FT-IR spectra for MWCNT/PIs and MWCNT- $g$-PIs. All the samples exhibited characteristic imide absorptions at around $1776 \mathrm{~cm}^{-1}$ (asymmetrical $\mathrm{C}=\mathrm{O}$ stretching), $1714 \mathrm{~cm}^{-1}$ (symmetrical $\mathrm{C}=\mathrm{O}$ stretching), and $1366 \mathrm{~cm}^{-1}$ (C-N stretching). The spectra of MWCNT- $g$-PIs show the asymmetrical and symmetrical stretching vibrations of $-\mathrm{CH}_{2}$ at $2921 \mathrm{~cm}^{-1}$ and $2846 \mathrm{~cm}^{-1}$, respectively. These vibrations belonged to APTES and carbon nanotubes, and no existence of the characteristic absorption bands of the $-\mathrm{NH}_{2}$ and $-\mathrm{OH}$ groups proved the successful

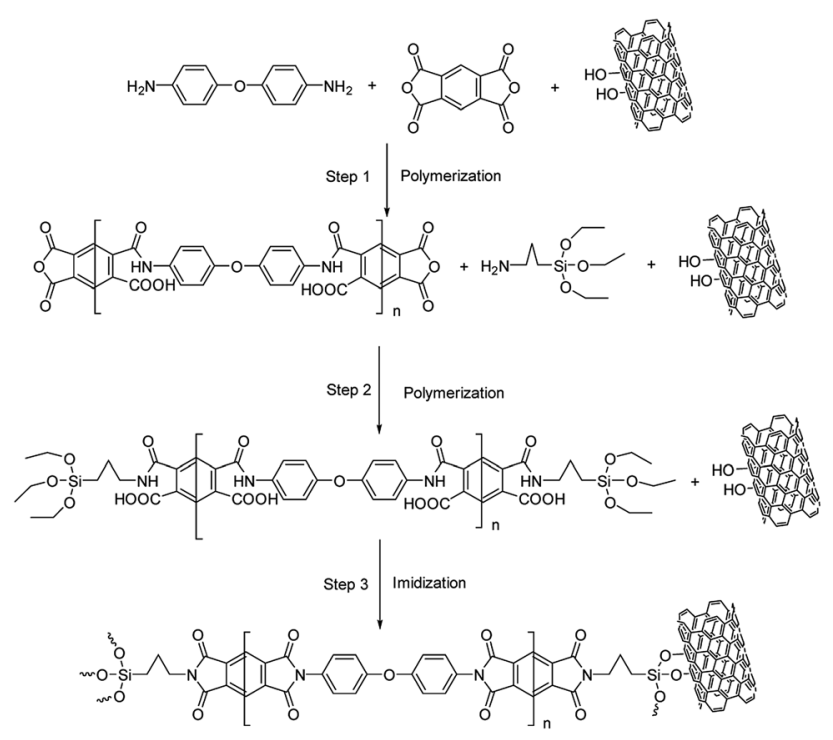

Scheme 1 The preparation process of MWCNT-g-PIs. 

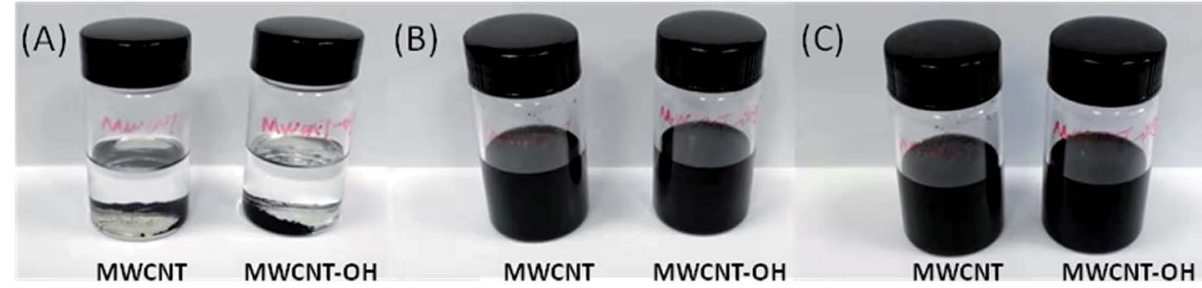

(D)

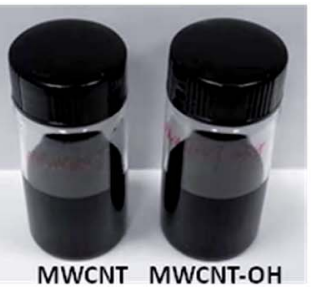

Fig. 1 The dispersed state of carbon nanotubes in $1 \mathrm{mg} \mathrm{mL}^{-1}$ DMAc: (A) before sonication; (B) after sonication for $3 \mathrm{~h}$; (C) $12 \mathrm{~h}$ after sonication; (D) $24 \mathrm{~h}$ after sonication.

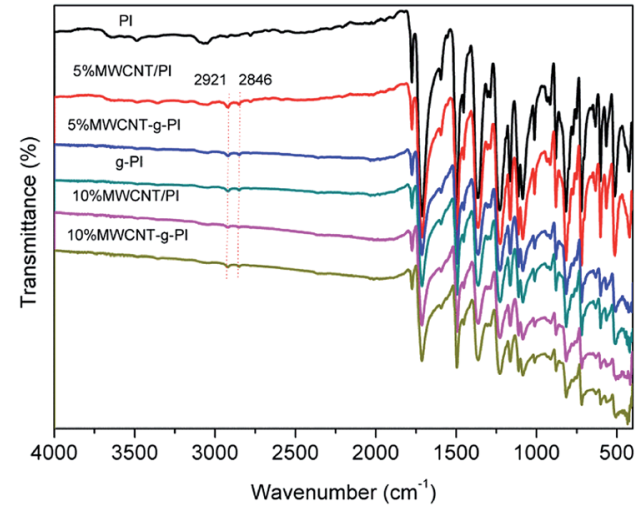

Fig. 2 FT-IR spectra of MWCNT/PIs and MWCNT-g-PIs.

grafting of polyimide chains on carbon nanotubes. The interaction between MWCNT and PI in MWCNT- $g$-PIs via coupling is illustrated in Fig. 3.

Fig. 4 shows the wide-angle X-ray diffraction (XRD) curves of MWCNT/PIs and MWCNT- $g$-PIs. PI and $g$-PI only exhibited a diffuse peak at $2 \theta=17.5^{\circ}$, whereas $5 \%$ and $10 \%$ MWCNT/PIs and MWCNT- $g$-PIs exhibited two diffuse peaks at $2 \theta=17.3^{\circ}$ and $24.9^{\circ}$, respectively. A small diffuse peak at $2 \theta=24.9^{\circ}$ was observed in the diffraction curves of $5 \%$ and $10 \%$ MWCNT/PIs

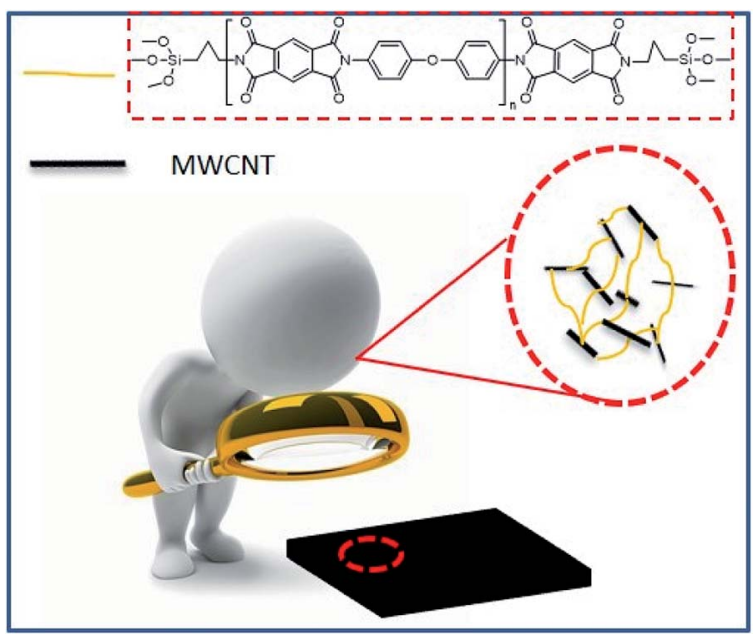

Fig. 3 Illustration of the interaction between MWCNTs and polyimide in MWCNT-g-PIs. and MWCNT- $g$-PIs, indicating that the carbon nanotubes were successfully incorporated into the polyimide matrix.

\subsection{Thermal properties of MWCNT/PIs and MWCNT- $g$-PIs}

Fig. 5 and 6 represent the thermal stability of MWCNT/PIs and MWCNT- $g$-PIs investigated by TGA under $\mathrm{N}_{2}$ and air atmospheres at the heating rate of $10^{\circ} \mathrm{C} \mathrm{min}^{-1}$; the results are listed in Table 1 . The $T_{5 \%}$ and $T_{10 \%}$ values of PI were $559^{\circ} \mathrm{C}$ and $573{ }^{\circ} \mathrm{C}$ under $\mathrm{N}_{2}$ atmosphere, respectively. Compared with the values for PI, the $T_{5 \%}$ and $T_{10 \%}$ of $g$-PI decreased slightly under $\mathrm{N}_{2}$ atmosphere; the values were $549{ }^{\circ} \mathrm{C}$ and $569{ }^{\circ} \mathrm{C}$, respectively. However, the addition of carbon nanotubes improved $T_{5 \%}$ and $T_{10 \%}$ under $\mathrm{N}_{2}$ atmosphere irrespective of whether by blending or grafting. By the thermal degradation curves of MWCNTs and MWCNT-OH under $\mathrm{N}_{2}$ atmosphere, we can infer that MWCNTs and MWCNT-OH have better thermal stability than PI, which results in the enhancement of $T_{5 \%}$ and $T_{10 \%}$ of the materials. The residual weight retentions at $800{ }^{\circ} \mathrm{C}$ also improved under $\mathrm{N}_{2}$ atmosphere; the values for $10 \%$ MWCNT/PI and $10 \%$ MWCNT- $g$-PI were $62.5 \%$ and $62.6 \%$, respectively. In contrast to the values for PI, $T_{5 \%}$ and $T_{10 \%}$ had a marked decrease under air atmosphere for the materials prepared by the blending method. From the DTG curves of MWCNT/PIs in air, we can infer that the degradation of MWCNTs at a high-temperature stage is the main reason for the above-mentioned phenomenon. However, $T_{5 \%}$ and $T_{10 \%}$ had a marked increase under air atmosphere for the materials prepared by the grafting method than the results obtained for the blending method. After grafting, MWCNTs were tightly wrapped by polyimide chains owing to the covalent

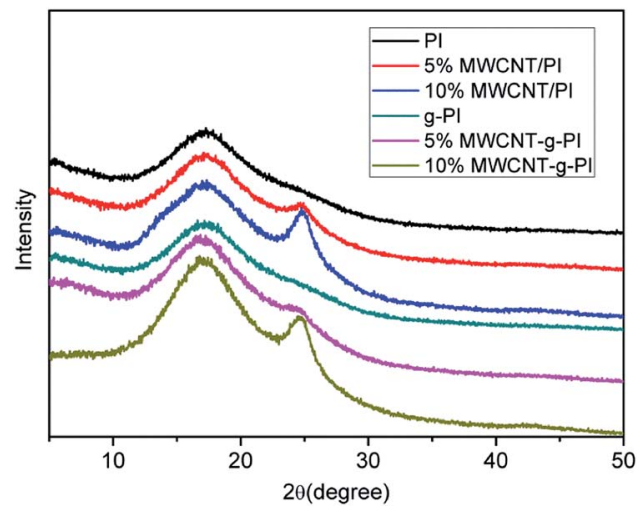

Fig. 4 XRD spectra of MWCNT/PIs and MWCNT-g-PIs. 

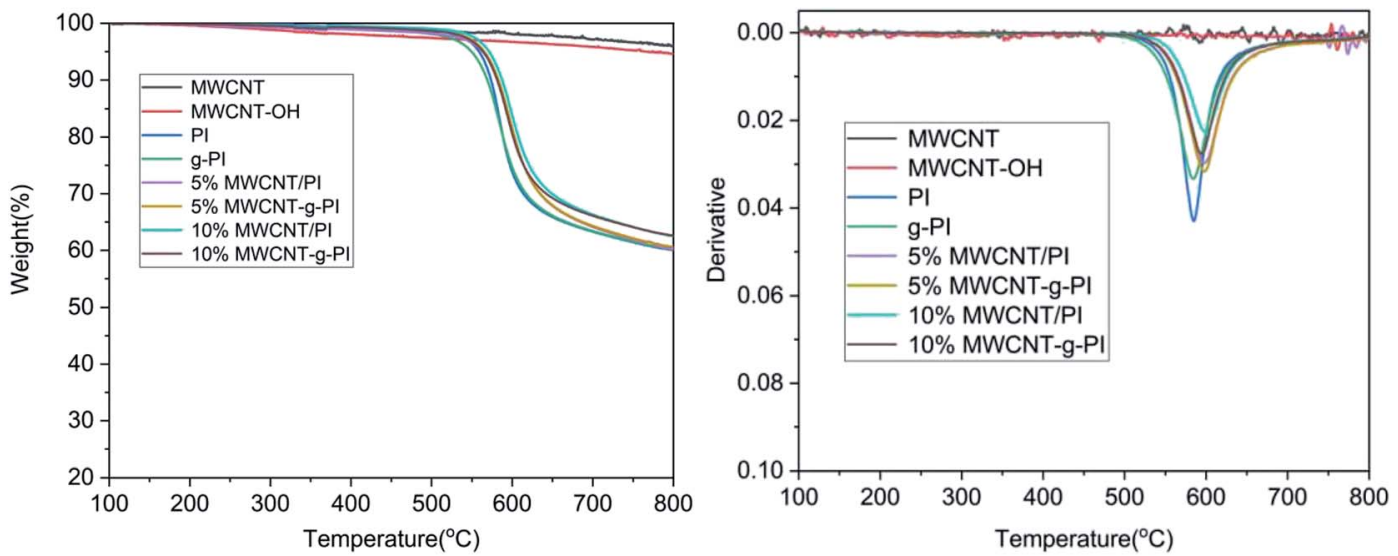

Fig. 5 TGA and DTG curves of MWCNTs, MWCNT-OH, MWCNT/PIs and MWCNT-g-PIs in $\mathrm{N}_{2}$.

bond linkage between MWCNTs and polyimide chains, which strengthened the interfacial interaction and thus, the MWCNT degradation was delayed. The heat-resistance index $\left(T_{\mathrm{HRI}}\right)$ was calculated; ${ }^{11,20}$ the results are listed in Table $1 . \mathrm{In} \mathrm{N}_{2}$, the $T_{\mathrm{HRI}}$ values of MWCNT/PIs and MWCNT-g-PIs increased after the addition of MWCNTs. Under air atmosphere, the $T_{\mathrm{HRI}}$ of MWCNT/PIs decreased after the addition of MWCNTs, but $T_{\mathrm{HRI}}$ of the MWCNT- $g$-PIs brought into correspondence with that of pure PI. In short, MWCNT/PIs and MWCNT-g-PIs exhibited good thermal stability in $\mathrm{N}_{2}$. However, MWCNT- $g$-PIs possessed better thermal stability than MWCNT/PIs in air. Nevertheless, the reduction in thermal stability for MWCNT/PIs in air was retained at an acceptable degree.

The dynamic mechanical analyses of MWCNT/PIs and MWCNT-g-PIs are shown in Fig. 7 and 8. The storage modulus retentions of MWCNT/PIs and MWCNT-g-PIs at $200{ }^{\circ} \mathrm{C}$ and $300{ }^{\circ} \mathrm{C}$ were analysed and listed in Table 2 . All the samples had good storage modulus retention at a high-temperature stage. The storage modulus retentions were greater than $63 \%$ at $200{ }^{\circ} \mathrm{C}$ and $45 \%$ at $300{ }^{\circ} \mathrm{C}$. Meanwhile, the glass transition temperature $\left(T_{\mathrm{g}}\right)$ was analysed; it was determined by the peak temperature of the $\tan \delta$ curves and listed in Table $1 . T_{\mathrm{g}}$ is possibly determined by two competitive factors: the free volume and the steric effect. ${ }^{21,22}$ In the MWCNT-g-PI system, $T_{g}$ shows a decreasing trend with the increase in the loading amount of MWCNTs. The polyimide chains grafted on the MWCNT surfaces disrupted the ordered chain structure of the polyimides and resulted in the increase in free volume. However, the $T_{\mathrm{g}}$ values of all the samples were greater than $399^{\circ} \mathrm{C}$.

\subsection{Mechanical properties of MWCNT/PIs and MWCNT- $g$ - PIs}

For the nanocomposites, the mechanical property is affected by many factors, such as the polymer matrix, loading amount of inorganic nanofillers, dispersion in the polymer matrix and interfacial interaction. ${ }^{23}$ Based on the several aspects mentioned above, the mechanical properties of MWCNT/PIs and MWCNT- $g$-PIs were discussed. The tensile strength, tensile modulus and elongation at break results of MWCNT/PIs and MWCNT- $g$-PIs are summarized in Table 3. The tensile strength, tensile modulus and elongation at break of PI were $129 \mathrm{MPa}, 2.39 \mathrm{GPa}$ and $57.5 \%$, respectively. PI showed good mechanical properties. Compared with the results for PI, the tensile strength and tensile modulus of $g$-PI had a slight increase because of the existence of crosslinking points by the self-polycondensation of the coupling agent at the ending of the
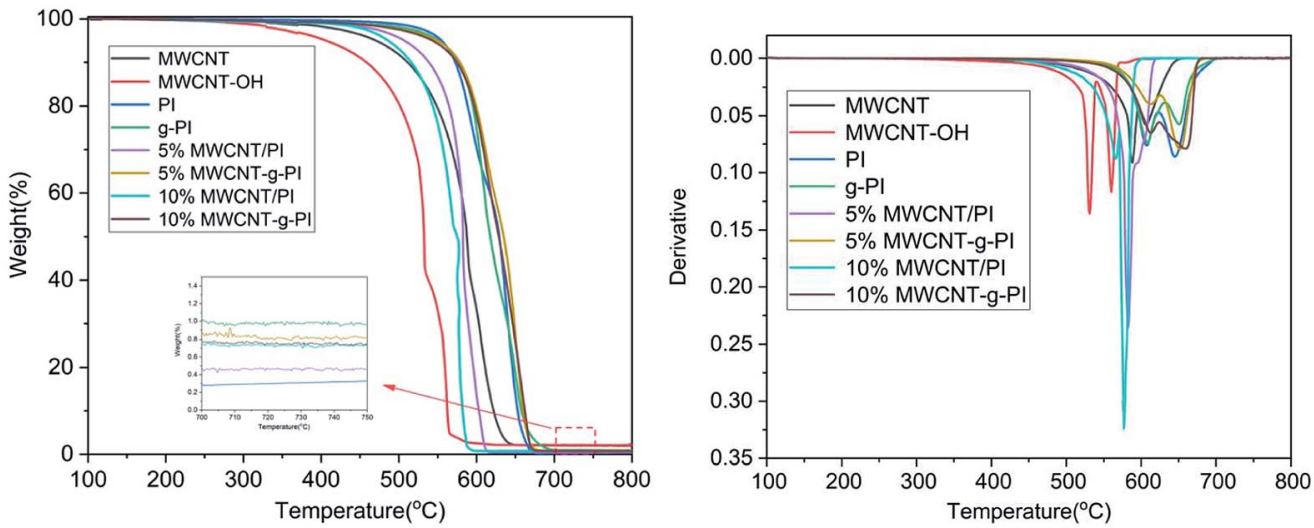

Fig. 6 TGA and DTG curves of MWCNTs, MWCNT-OH, MWCNT/PIs and MWCNT-g-PIs in air. 
Table 1 Thermal properties of MWCNT/PIs and MWCNT-g-PIs

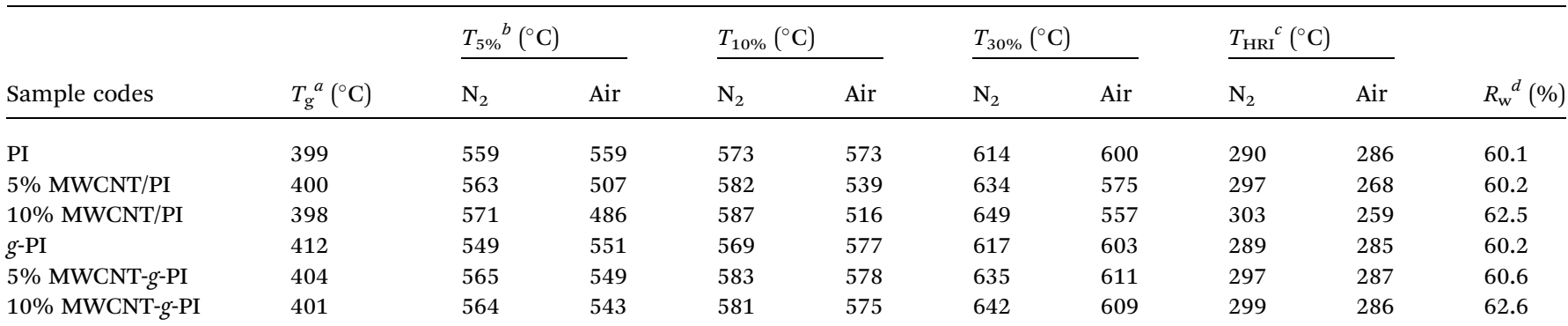

${ }^{a}$ Measured by DMA at a heating rate of $5{ }^{\circ} \mathrm{C} \mathrm{min}^{-1} .{ }^{b} 5 \%$ weight loss temperature $\left(T_{5 \%}\right)$ and $10 \%$ weight loss $\left(T_{10}\right)$ temperature measured by TGA.

${ }^{c}$ Heat-resistance index $\left(T_{\mathrm{HRI}}\right)$ was calculated by the equation $T_{\mathrm{HRI}}=0.49 \times\left[T_{5 \%}+0.6 \times\left(T_{30 \%}-T_{5 \%}\right)\right]$. ${ }^{d}$ Residual weight retention at $800{ }^{\circ} \mathrm{C}$.

polyimide chains, which was also responsible for the decrease in the elongation at break of $g$-PI from $57.5 \%$ to $48.6 \%$. Subsequently, the mechanical properties of MWCNT- $g$-PIs with different loading amounts were analysed. $5 \%$ and $10 \%$ MWCNT- $g$-PIs maintained the high tensile strength of PI. The tensile modulus increased up to $2.59 \mathrm{GPa}$ on increasing the

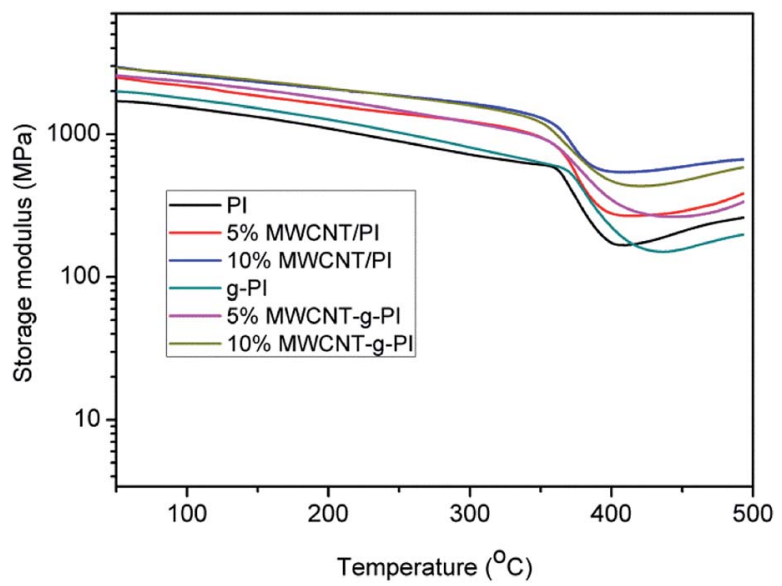

Fig. 7 Storage modulus curves of MWCNT/PIs and MWCNT-g-PIs.

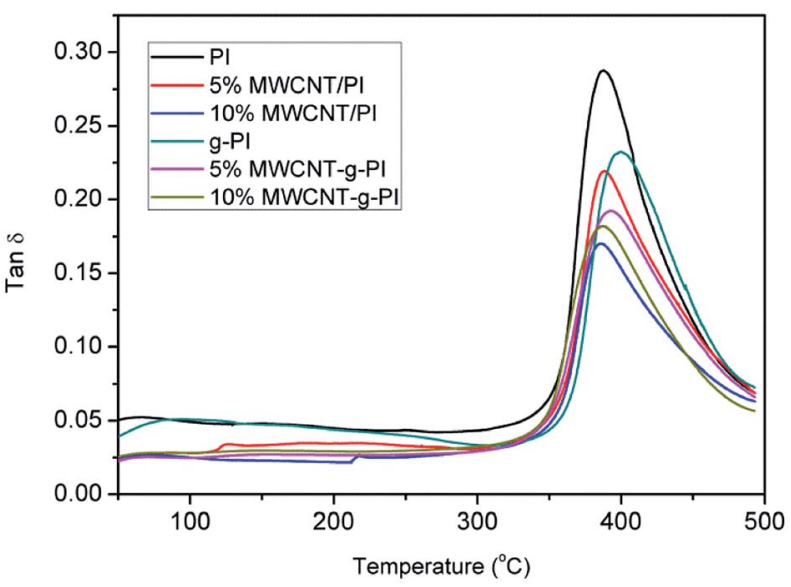

Fig. $8 \tan \delta$ curves of MWCNT/PIs and MWCNT-g-Pls.
MWCNT loading. The elongation at break of MWCNT- $g$-PIs exhibited a reducing trend but was still more than $36 \%$. The reduction in the elongation at break in our system was retained at an acceptable degree. The covalent bond linkage between MWCNTs and polyimide chains promoted the well-distributed dispersion of MWCNTs in polyimides and strengthened the interfacial interaction between MWCNTs and polyimide chains. Hence, MWCNT- $g$-PIs showed good mechanical properties. The mechanical properties of the sample prepared by the simple blending method (MWCNT/PIs) were also investigated. In this research, a short carbon nanotube $(L / d=250)$ was selected, which could be easily dispersed in a polymer matrix and lead to the existence of $\pi-\pi$ interactions between the carbon

Table 2 Storage modulus retention of MWCNT/PIs and MWCNT-gPls decided by storage modulus curves ${ }^{a}$

\begin{tabular}{llc}
\hline & \multicolumn{2}{l}{ Storage modulus retention $(\%)$} \\
\cline { 2 - 3 } Sample codes & $200{ }^{\circ} \mathrm{C}$ & $300{ }^{\circ} \mathrm{C}$ \\
\hline PI & 68.0 & 53.4 \\
$5 \%$ MWCNT/PI & 70.2 & 52.4 \\
$10 \%$ MWCNT/PI & 72.4 & 53.4 \\
$g$-PI & 63.9 & 45.8 \\
$5 \%$ MWCNT- $g$-PI & 68.3 & 50.8 \\
$10 \%$ MWCNT- $g$-PI & 67.1 & 50.1 \\
${ }^{a}$ Storage modulus retention $(\%)$ & $\frac{\text { storage modulus }(T)}{\text { storage modulus }\left(T_{50}\right)} \times 100 \%$.
\end{tabular}

Table 3 Mechanical properties of MWCNT/PIs and MWCNT-g-PIs

\begin{tabular}{llll}
\hline Sample codes & $T_{\mathrm{S}}{ }^{a}(\mathrm{MPa})$ & $T_{\mathrm{M}}{ }^{b}(\mathrm{GPa})$ & $E_{\mathrm{B}}{ }^{c}(\%)$ \\
\hline PI & 129 & 2.38 & 57.5 \\
$5 \%$ MWCNT/PI & 132 & 2.39 & 53.6 \\
$10 \%$ MWCNT/PI & 128 & 2.70 & 41.8 \\
$g$-PI & 132 & 2.39 & 48.6 \\
$5 \%$ MWCNT- $g$-PI & 124 & 2.41 & 36.6 \\
$10 \%$ MWCNT- $g$-PI & 129 & 2.59 & 36.1
\end{tabular}

${ }^{a} T_{\mathrm{S}}$, tensile strength. ${ }^{b} T_{\mathrm{M}}$, tensile modulus. ${ }^{c} E_{\mathrm{B}}$, elongation at break. 

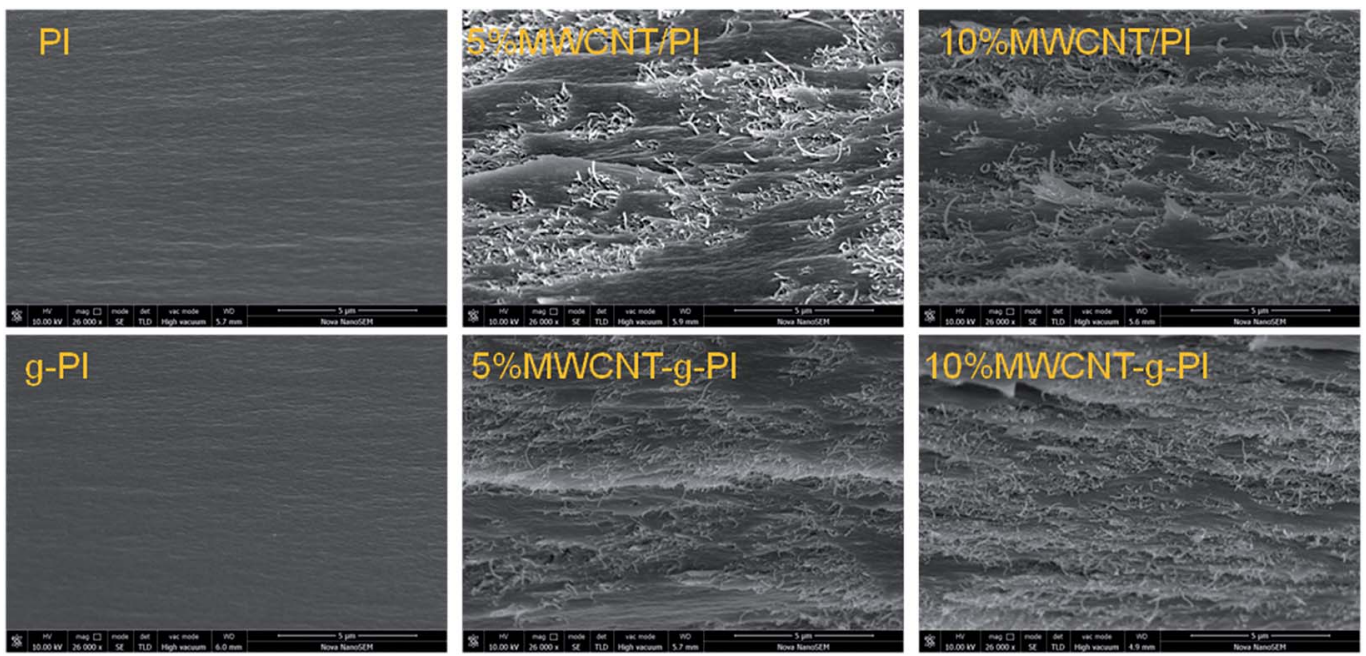

Fig. 9 Cross-section morphology of MWCNT/PIs and MWCNT-g-PIs.

nanotubes and benzene rings in polyimide chains. Thus, MWCNT/PIs also exhibited good mechanical properties.

\subsection{Morphology of MWCNT/PIs and MWCNT- $g$-PIs}

Fig. 9 exhibits the SEM images of MWCNT/PIs and MWCNT-gPIs. In Fig. 9, it can also be noticed that the MWCNTs disperse more homogeneously in MWCNT-g-PIs than in MWCNT/PIs due to covalent bond linkage, strengthening the interfacial interaction between MWCNTs and the polyimide matrix. A small portion of agglomerated MWCNTs can be seen in the 5\% and $10 \%$ MWCNT/PI composites. This is one of the key factors that can affect the thermal conductivity of the resulting composites. Apparently, a higher filler content is required to form "thermal conductive pathways" when the fillers agglomerate in the polymer matrix. A good dispersion of MWCNTs in polyimides may contribute to the improvement in thermal conductivity.

\subsection{Thermal conductivity of MWCNT/PIs and MWCNT- $g$-PIs}

The thermal conductivity properties of the MWCNT/PIs and MWCNT-g-PI composites are shown in Fig. 9. The increasing
MWCNT loading enhanced the thermal conductivity of MWCNT/PIs and MWCNT- $g$-PIs because more and more MWCNTs participated in forming "thermal conductive pathways". However, the thermal conductivity of MWCNT-g-PIs increased faster than that of MWCNT/PIs at the same loading. The thermal conductivity of $10 \%$ MWCNT/PIs improved by $69.6 \%$ than that of pure PI. The thermal conductivity of $10 \%$ MWCNT- $g$-PIs increased by $87.0 \%$ than that of pure PI (Fig. 10). The well-distributed dispersion of MWCNTs in polyimides can be boosted to form "thermal conductive pathways" at the same loading, and strengthening the interfacial interaction between MWCNTs and polyimide chains by covalent bond linkage can decrease the interfacial thermal resistance $\left(R_{\mathrm{C}}\right)$ between nanotubes and the polymer matrix.

The interfacial thermal resistance $\left(R_{\mathrm{C}}\right)$ was calculated by the Maxwell-Garnett-type effective medium approach (EMA) in our research as follows: ${ }^{24}$

$$
K=K_{\mathrm{m}} \frac{3+f\left(\beta_{x}+\beta_{z}\right)}{3-f \beta_{x}}
$$
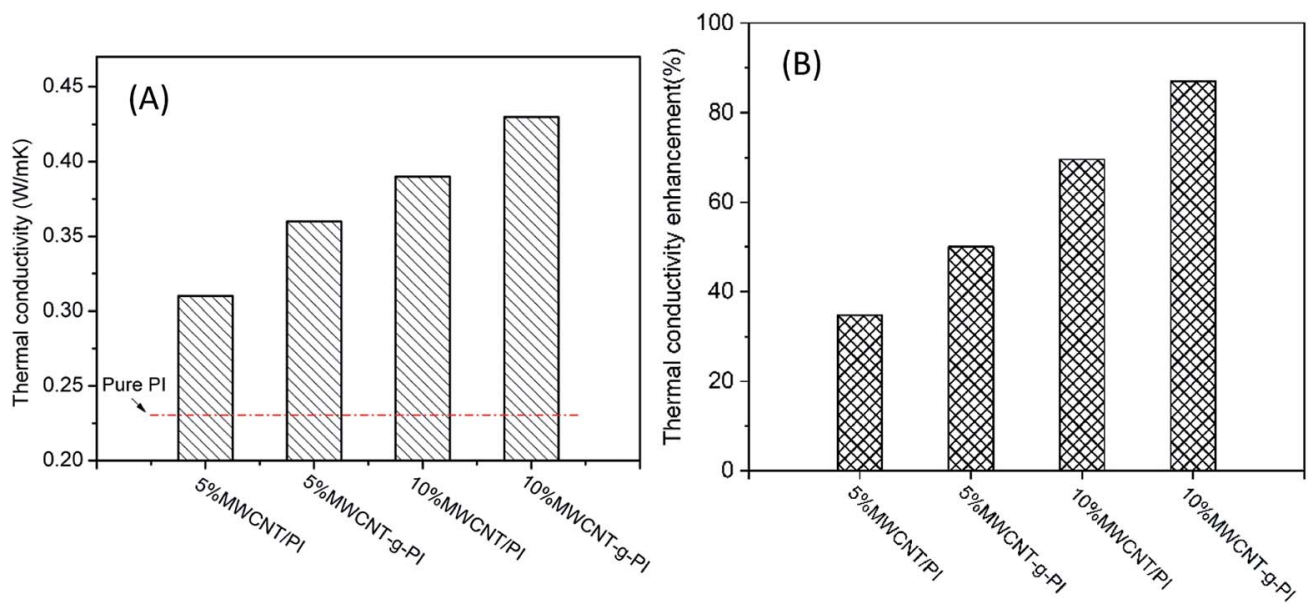

Fig. 10 Thermal conductivity (A) and thermal conductivity enhancement (B) of MWCNT/PIs and MWCNT-g-PIs. 
Table 4 Thermal conductivity of MWCNT/polymer composites listed in literature compared with the results of this work

\begin{tabular}{llll}
\hline Composites & Loading $(\mathrm{wt} \%)$ & Thermal conductivity $\left(\mathrm{W} \mathrm{m}^{-1} \mathrm{~K}^{-1}\right)$ & Year and ref \\
\hline MWCNT/poly(ether ether ketone) & 17 & 0.70 & 2009 (ref. 25) \\
MWCNT/expoxy & 10 & 0.35 & 2012 (ref. 16) \\
MWCNT/phenoxy & 10 & 0.42 & 2013 (ref. 26) \\
MWCNT/poly(ether sulfone) & 10 & 0.35 & 2013 (ref. 26) \\
MWCNT/polyamide-6 & 4 & 0.28 & 2009 (ref. 27) \\
MWCNT/polyimide & 3 & 0.25 & 2019 (ref. 3) \\
MWCNT/expoxy & 2 & 0.25 & 2019 (ref. 5) \\
MWCNT-graft-polyimides & 5 & 0.36 & This work
\end{tabular}

$$
\beta_{x}=\frac{2\left(K_{11}^{\mathrm{c}}-K_{\mathrm{m}}\right)}{K_{11}^{\mathrm{c}}+K_{\mathrm{m}}}, \beta_{z}=\frac{K_{33}^{\mathrm{c}}}{K_{\mathrm{m}}}-1
$$

Here, $f$ is the volume fraction of the carbon nanotubes, $K_{\mathrm{m}}$ represents the thermal conductivity of PI $\left(0.23 \mathrm{~W} \mathrm{~m}^{-1} \mathrm{~K}^{-1}\right), K_{\mathrm{c}}$ represents the thermal conductivity of MWCNTs $\left(3000 \mathrm{~W} \mathrm{~m}^{-1}\right.$ $\mathrm{K}^{-1}$ ), $K_{11}^{\mathrm{c}}$ represents the equivalent thermal conductivities along transverse and $K_{33}^{\mathrm{c}}$ represents the longitudinal axes of the composite unit cell. $K_{11}^{\mathrm{c}}$ and $K_{33}^{\mathrm{c}}$ can be expressed as follows:

$$
K_{11}^{\mathrm{c}}=\frac{K_{\mathrm{c}}}{1+\frac{2 a_{\mathrm{K}} K_{\mathrm{c}}}{d K_{\mathrm{m}}}}, K_{33}^{\mathrm{c}}=\frac{K_{\mathrm{c}}}{1+\frac{2 a_{\mathrm{K}} K_{\mathrm{c}}}{L K_{\mathrm{m}}}}
$$

Here, $d$ represents the diameter of the carbon nanotubes $(d=8$ $\mathrm{nm})$ and $L$ represents the length of the carbon nanotubes $(L=2$ $\mu \mathrm{m}) ; a_{\mathrm{K}}$ is the so-called Kapitza radius, which is expressed as follows:

$$
a_{\mathrm{K}}=R_{\mathrm{C}} K_{\mathrm{m}}
$$

According to the above-mentioned equations, the interfacial thermal resistance $\left(R_{\mathrm{C}}\right)$ of $3.0 \times 10^{-8} \mathrm{~m}^{2} \mathrm{~K} \mathrm{~W}^{-1}$ for the $10 \%$ MWCNT/PI composite and $2.5 \times 10^{-8} \mathrm{~m}^{2} \mathrm{~K} \mathrm{~W}^{-1}$ for the $10 \%$ MWCNT- $g$-PI composite was observed. In contrast to the value for $10 \% \mathrm{MWCNT} / \mathrm{PI}$, the interfacial thermal resistance $\left(R_{\mathrm{C}}\right)$ of $10 \%$ MWCNT- $g$-PI decreased by $16.7 \%$, which was consistent with the results we expected. Strengthening the interfacial interaction between MWCNTs and polyimide chains is necessary to reduce the interfacial thermal resistance $\left(R_{\mathrm{C}}\right)$ and then improve the thermal conductivity.

The thermal conductivity data of MWCNT-graft-polyimides were compared with that reported for MWCNT/polymer composites in the current literature; the results are listed in Table 4. The data showed that the thermal conductivity of MWCNT-graft-polyimides was higher than that of most MWCNT/polymer composites reported previously. Compared with the thermal conductivity of $10 \% \mathrm{MWCNT} / \mathrm{PI}$, the thermal conductivity of $10 \%$ MWCNT- $g$-PI increased by $10.3 \%$.

\section{Conclusions}

In this paper, it was found that MWCNT- $g$-PIs possessed higher thermal conductivity than MWCNT/PIs. In contrast to the interfacial thermal resistance of $10 \%$ MWCNT/PIs, the interfacial thermal resistance of $10 \%$ MWCNT- $g$-PI decreased by 16.7\%. Meanwhile, MWCNT/PIs and MWCNT-g-PIs showed good thermal and mechanical properties. The $T_{\mathrm{g}}$ values of all the samples were greater than $399{ }^{\circ} \mathrm{C}$. The thermal stability of $5 \%$ and $10 \%$ MWCNT- $g$-PIs in air was enhanced compared to that for $5 \%$ and $10 \%$ MWCNT/PIs. The storage modulus retentions were greater than $63 \%$ at $200{ }^{\circ} \mathrm{C}$ and $45 \%$ at $300{ }^{\circ} \mathrm{C}$. $5 \%$ and $10 \%$ MWCNT- $g$-PIs maintained the high tensile strength of PI. The tensile modulus increased up to $2.59 \mathrm{GPa}$ with the increase in the MWCNT loading. This work provides a guideline for improving the thermal conductivity of polyimides effectively at relatively low loadings.

\section{Conflicts of interest}

There are no conflicts to declare.

\section{References}

1 K. Sato, H. Horibe, T. Shirai, Y. Hotta, H. Nakano, H. Nagai and K. Mitsuishi, J. Mater. Chem., 2010, 20(14), 2749.

2 D. Ding, H. Wang, Z. Wu, Y. Chen and Q. Zhang, Macromol. Rapid Commun., 2019, 40, 1800805.

3 W. Yan, Y. Zhang, H. Sun, S. Liu, Z. Chi, X. Chen and J. Xu, J. Mater. Chem. A, 2014, 2(48), 20958-20965.

4 C. Liang, P. Song, A. Ma, X. Shi, H. Gu, L. Wang, H. Qiu, J. Kong and J. Gu, Compos. Sci. Technol., 2019, 181, 107683. 5 X. Wang, W. Li, Z. Zhang, K. Chen and W. Gan, J. Appl. Polym. Sci., 2019, 136(35), 47911.

6 Y. Hwang, M. Kim and J. Kim, Composites, Part A, 2013, 55, 195-202.

7 S. Y. Yang, W.-N. Lin, Y.-L. Huang, H.-W. Tien, J.-Y. Wang, C.-C. M. Ma, S.-M. Li and Y.-S. Wang, Carbon, 2011, 49(3), 793-803.

8 Y. Sun, L. Chen, J. Lin, P. Cui, M. Li and X. Du, J. Compos. Mater., 2017, 51(12), 1743-1752.

9 C. C. Teng, C.-C. M. Ma, C.-H. Lu, S.-Y. Yang, S.-H. Lee, M.-C. Hsiao, M.-Y. Yen, K.-C. Chiou and T.-M. Lee, Carbon, 2011, 49(15), 5107-5116. 
10 Y. Guo, X. Yang, K. Ruan, J. Kong, M. Dong, J. Zhang, J. Gu and Z. Guo, ACS Appl. Mater. Interfaces, 2019, 11, 2546525473.

11 X. Yang, S. Fan, Y. Li, Y. Guo, Y. Li, K. Ruan, S. Zhang, J. Zhang, J. Kong and J. Gu, Composites, Part A, 2020, 128, 105670.

12 Y. Guo, Z. Lyu, X. Yang, Y. Lu, K. Ruan, Y. Wu, J. Kong and J. Gu, Composites, Part B, 2019, 164, 732-739.

13 S. Choi and J. Kim, Composites, Part B, 2013, 51, 140-147.

14 S. Z. Yu, P. Hing and X. Hu, Composites, Part A, 2002, 33, 289292.

15 X. Wu, P. Jiang, Y. Zhou, J. Yu and Y. Yin, J. Appl. Polym. Sci., 2014, 131(15), 40528.

16 J. G. Park, Q. Cheng, J. Lu, J. Bao, S. Li, Y. Tian, Z. Liang, C. Zhang and B. Wang, Carbon, 2012, 50, 2083-2090.

17 A. Wurm, D. Lellinger, A. A. Minakov, T. Skipa, P. Pötschke, R. Nicula, I. Alig and C. Schick, Polymer, 2014, 55(9), 22202232.

18 H. Im and J. Kim, J. Mater. Sci., 2012, 47(16), 6025-6033.
19 B. Tonpheng, J. Yu and O. Andersson, Macromolecules, 2009, 42(23), 9295-9301.

20 Y. Han, X. Shi, X. Yang, Y. Guo, J. Zhang, J. Kong and J. Gu, Compos. Sci. Technol., 2020, 187, 107944.

21 G. Ragosta, P. Musto, M. Abbate and G. Scarinzi, Polymer, 2009, 50(23), 5518-5532.

22 A. Strachota, I. Kroutilova, J. Kovarova and L. Matejka, Macromolecules, 2004, 37, 9457-9464.

23 G. Zhi, M. Huo, J. Mu, S. Zhang, Y. Lu, J. Luan, P. Huo, Y. Du and G. Wang, J. Mater. Chem. C, 2014, 2, 1094-1103.

24 T. C. Clancy and T. S. Gates, Polymer, 2006, 47(16), 59905996.

25 D. S. Bangarusampath, H. Ruckdäschel, V. Altstädt, J. K. W. Sandler, D. Garray and M. S. P. Shaffer, Polymer, 2009, 50, 5803-5811.

26 J. Bouchard, A. Cayla, E. Devaux and C. Campagne, Compos. Sci. Technol., 2013, 86, 177-184.

27 Z. Shen, S. Bateman, D. Y. Wu, P. McMahon, M. Dell'Olio and J. Gotama, Compos. Sci. Technol., 2009, 69, 239-244. 